\title{
Screening of dystrophin gene deletions in Egyptian patients with DMD/BMD muscular dystrophies
}

\author{
Laila K. Effat ${ }^{\mathrm{a}}$, Ashraf A. El-Harouni ${ }^{\mathrm{a}}$, \\ Khalda S. Amr ${ }^{\mathrm{a}}$, Tarik I. El-Minisi ${ }^{\mathrm{b}}$, \\ Nagwa Abdel Meguid ${ }^{\mathrm{a}}$ and Mostafa El-Awady ${ }^{\mathrm{a}}$ \\ ${ }^{a}$ Department of Human Genetics, National Research \\ Center, Dokki, Cairo 12311, Egypt \\ ${ }^{\mathrm{b}}$ Neurology Department, Faculty of Medicine, \\ Al-Azhar University, Egypt
}

Received 20 November 1999

Accepted 25 April 2000

Duchenne muscular dystrophy (DMD) and Becker muscular dystrophy (BMD) are allelic disorders caused by mutations within the dystrophin gene. Our study has identified 100 Egyptian families collected from the Human Genetics Clinic, National Research Center, Cairo. All cases were subjected to complete clinical evaluation pedigree analysis, electromyography studies, estimation of serum creatine phosphokinase enzyme (CPK) levels and DNA analysis. Multiplex PCR using 18 pairs of specific primers were used for screening of deletion mutations within the dystrophin gene. A frequency of $55 \%$ of deletions were found among the families. Sixty per cent of detected deletions involved multiple exons spanning the major or the minor hot spot of the dystrophin gene. The remainder $40 \%$ involved single exon deletions, which mainly involved exon 45. Comparing these findings with frequencies of other countries it was found that our figures fall within the reported range of $40 \%-60 \%$ for deletions. The distribution of deletions in our study and other different studies was variable and specific ethnic differences do not apparently account for specific deletions. In addition this study concluded that employment of the 18 exon analysis is a cost effective and a highly accurate (97\% detection rate) method to be considered when planning to launch a nationwide program.

\section{Introduction}

DMD and BMD are variable phenotypic expressions of the dystrophin gene defect at the Xp21 site on the human X chromosome. DMD has a worldwide distribu- tion with a mean incidence of 1 : 3500 male births [11]. The phenotypic differences between the two dystrophies is that BMD has a later age of onset, unassisted ambulation after age 15 , and a later survival rate into adult life. Most children with DMD have gait disturbance before the age of 5 and become bound to a wheelchair before the age of 10 [5]. DMD/BMD are caused by mutations in the human dystrophin gene that is $2.4 \mathrm{Mbp}$ in size and comprises 79 exons [18]. Approximately $2 / 3$ of the boys affected with DMD/ BMD have deletion mutations of one or more exons within the dystrophin gene [4]. Previous studies demonstrated that although dystrophin deletions are heterogeneous and vary in size and locations they are clustered in two hot spots; the major site encompassing exons 45-52, and the minor region including exons 3-19 [16]. In some DMD patients such mutations cause deletions in functional domains while in others they induce premature termination of protein translation with a resultant deficiency of functional dystrophin [12]. The phenotype largely depends on whether the deletion disrupts the reading frame of the dystrophin gene and results in virtually no dystrophin. In BMD patients mutations do not disrupt the reading frame and result in an abnormal but partially functional dystrophin [15]. This hypothesis holds true for more than $90 \%$ of cases. Exceptions such as mild BMD with a frameshift deletion can be explained by reinitiating transcription from an internal start codon inframe mutation involving the central rod domain. This can result in a very mild weakness, myalgia alone or even asymptomatic CK-emia. In contrast, deletions resulting in a deficiency of the C-terminal region of dystrophin result in a severely diseased phenotype [6].

In this study we scanned the dystrophin gene for deletion mutations at 18 exons clustered within the minor and major hot spots in patients with DMD/BMD. The aim of this study is to identify the genetic background of different types of deletion mutations and to corre- 
late the incidence of these deletions with international figures for determination of ethnic differences.

\section{Materials and methods}

This study included 100 families with 165 DMD/ BMD male patients. Their age ranged from 3 to 40 years. The patients were referred to the Human Genetics Clinic (National Research Center, Cairo, Egypt) for genetic counseling. The diagnosis was established in all cases on the bases of clinical and neurological evaluation, complete pedigree history and serum creatine phosphokinase levels.

Genomic DNA was extracted from peripheral blood leukocytes of 100 index cases by salting out method described by Miller et al. [14]. Amplification by Polymerase Chain Reaction (PCR) of 18 exons was carried out in six multiplex reactions using three pairs of primers with matched melting temperatures for each reaction. Each reaction produces three separable bands visualized on a $3 \%$ agarose ethidium stained gel. Exons studied were: Pm1, 3, 4, 6, 8, 12, 13, 17, 19, 43, $44,45,47,48,50,51,52,60$. The six multiplex PCR reactions were arranged as follows; reaction I: Pm, 50, 52; reaction II: 3,13, 47; reaction III: 43, 6, 60; reaction IV: $19,8,4$; reaction V: 45, 51, 44 and reaction VI: $48,17,12$. Thermal cycling profile for reactions I, II and III were [one cycle at $94^{\circ} \mathrm{C}$ for 6 minutes, $30 \mathrm{cy}$ cles each includes 30 seconds at $94^{\circ} \mathrm{C}$, one minute at $60^{\circ} \mathrm{C}, 4$ minutes at $65^{\circ} \mathrm{C}$ and one cycle at $65^{\circ} \mathrm{C}$ for 10 minutes]. For reactions IV, V and VI cycling conditions were [one cycle at $94^{\circ} \mathrm{C}$ for 6 minutes, 22 cycles each includes 30 seconds at $94^{\circ} \mathrm{C}, 30$ seconds at $53^{\circ} \mathrm{C}$, 4 minutes at $65^{\circ} \mathrm{C}$ and one cycle at $65^{\circ} \mathrm{C}$ for 7 minutes]. Primers were synthesized according to published sequences: exons Pm1, 3, 6, 13, 43, 47, 50, 52, 60 [1, 4], exons 4, 8, 19, 48, $51[1,8]$ and exons 12, 17, 44, $45[8]$.

\section{Results}

The diagnosis of DMD/BMD in the 100 male patients included in the study was established on clinical and neurological assessments. All patients had muscle wasting, weakness and diminished or absent tendon reflexes. 87/100 cases were diagnosed to have DMD and $13 / 100$ to have BMD. The average age at which patients become bound to wheelchairs was 12 years in DMD and 40 years in BMD patients. All patients had waddling gait, pseudohypertrophy of calf muscles, positive Gower's sign and exaggerated lumber lordosis. Myopathic EMG patterns and marked elevation of serum creatine phosphokinase level were constant findings in all cases. A hundred probands with DMD/BMD serving as index cases from 100 Egyptian families were examined for dystrophin gene deletions at 18 exons as described in "Materials and Methods".

Figure 1 illustrates the genetic deletions detected in $55 \%$ families (55/100). Forty-five families (45\%) had no detectable deletions in the dystrophin gene. The Single and multiple deletions were distributed as follows: 30 families had multiple deletion mutation and 25 families had single deletions. Deletions were clustered at either the major hot spot or at the minor hot spot of the gene.

Multiple deletions covering the major hot spot included 26/30 families at exons 45-52. Four multiple deletions (4/30) were found within the minor hotspot; del 6-17 (1/4), del 12-19 (1/4), del Pm \& del 3,13 (2/4).

Single exon deletions were identified in $45.5 \%$ of families $(25 / 55)$ of which $24 \%$ these deletions $(6 / 25)$ were found to be exon 45 .

Most single deletions were variable within the major hot spot and included exons 43(1/25), 44(2/25), $45(6 / 25), 47(4 / 25), 48(5 / 25), 50(4 / 25), 51(2 / 25)$. One family had a single minor deletion at exon 19 , the commonest single deletion within this group was exon 45 .

Figure 2 illustrates an example of the amplifications of 6 multiplex reactions in a patient with DMD, the mother and a normal control. The results displayed in the figure indicated a multiple deletion spanning exons 45 to 52 within the major hot spot.

Table 1 summarizes the presented data with the reported international studies.

\section{Discussion}

DMD/BMD muscular dystrophies are variable phenotype expression caused by mutations within the dystrophin gene. Elucidation of the molecular defects underlying genetic disorders is an indispensable step in the initiation of disease control programs in different countries. The phenotype largely depends on whether the mutation disrupts the reading frame of the gene (frame shift) leading to severe DMD or spares the reading frame (inframe) resulting in the milder form the BMD [7]. Based on the sequence information derived from published exon/intron junction multiple deletions covering exons $45-50$ as well as single deletions at ex- 


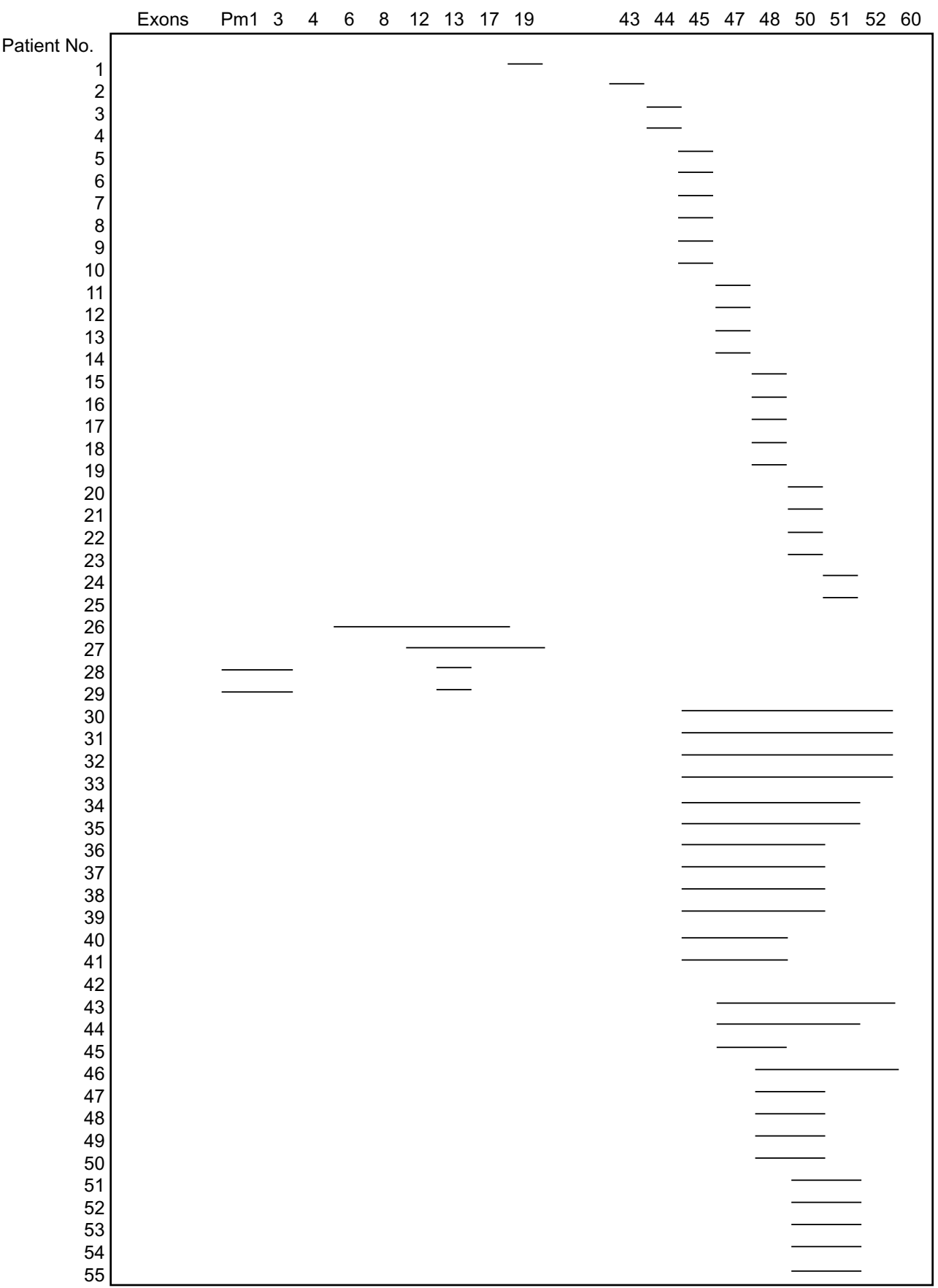

Fig. 1. Exon deletions of the dyrophin gene in 55 Egyptian families.

ons 45 and 50 are expected to disrupt the reading frame of the dystophin gene [13]. The majority of affected boys in this study $87 / 100$ were diagnosed clinically to have DMD and the rest 13/100 the milder form BMD.

The molecular analysis revealed either no deletion, multiple or single deletions within the major hotspot regarding DMD patients, deletions found in BMD were multiple or single but most probably inframe.

The development of multiplex PCR technique has permitted rapid and sensitive identification of up to $98 \%$ of dystrophin deletions [4]. This study screened the dystrophin deletions in attempt to shed light on 
Table 1

Comparison of frequency of deletions in different ethnic groups using multiplex PCR

\begin{tabular}{|c|c|c|c|c|c|}
\hline Authors & Country & Year & Exons tested & Deletions & $\begin{array}{c}\text { Deletions } \\
\% \text { at exons } 45-52\end{array}$ \\
\hline Tsukamoto et al. & Japan & 1991 & $4,8,12,17,19,44,45,48,51$ & $12 / 28(43 \%)$ & 75.1 \\
\hline Yang et al. & China & 1994 & $1,3,6,8,13,17,43,47,50,52,60$ & $15 / 41(37 \%)$ & Not mentioned \\
\hline Ballo et al. & S. Africa & 1994 & $3,4,6,8,13,19,42,44,45,48,50-53$ & $52 / 128(40 \%)$ & 65 \\
\hline Covone et al. & Italy & 1992 & $\begin{array}{l}\operatorname{Pm}(1), 3,4,6,8,12,13,16,17,19-22,29 \\
32,34,41-52,60\end{array}$ & $96 / 152(63 \%)$ & Not mentioned \\
\hline Niemann-Seyde et al. & Germany & 1992 & $\operatorname{Pm}(1), 3,4,6,8,12,13,17,19,43-52,60$ & $35 / 56(62.5)$ & 57.5 \\
\hline Baranov et al. & Russia & 1993 & $\operatorname{Pm}(1), 6,8,12,17,19,42-45,47,48,50,52$ & $49 / 119(41 \%)$ & 73.5 \\
\hline $\begin{array}{l}\text { Chamberlain et al. } \\
\text { (multicenter study) }\end{array}$ & US & 1992 & $4,8,17,19,44,45,48,51$ & $346 / 745(46 \%)$ & 69.7 \\
\hline Zatz et al. & Brazil & 1998 & $\begin{array}{l}\operatorname{Pm}(1), 3,4,6,8,12,13,17,19,43,44,45, \\
47,48,50,51,52,60\end{array}$ & $383 / 615(62.3)$ & Not mentioned \\
\hline Present study & Egypt & 1998 & $\begin{array}{l}\operatorname{Pm}(1), 3,4,6,8,12,13,17,19,43,44,45 \\
47,48,50,51,52,60\end{array}$ & $55 / 100(55 \%)$ & 14.5 \\
\hline
\end{tabular}

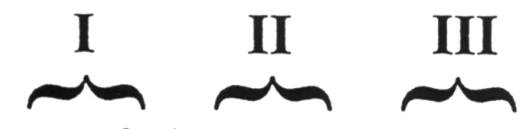

$\begin{array}{lllllllllll}1 & 2 & 3 & 4 & 5 & 6 & 7 & 8 & 9 & 10 & 11\end{array}$

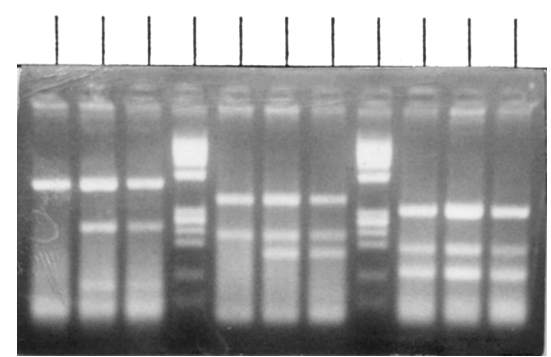

Fig. 2(a). Illustrates 3 multiplexes showing three bands each when normal. Multiplex I: one band and two absent bands (deletions: exon 50 and 52) in lane (1), three normal bands in lanes (2) and (3). Mutiplex II: two bands and one absent band (deletion exon 47) in lane (5), three normal bands in lanes (7) and (8). Multiplex lanes (7) and (8). Mutiplex III: three bands in lanes (9)-(11).

the Egyptian genetic background of DMD and BMD. The analysis involved 18 exons that were known to be clustered in the minor hot spot (exons Pm1, 3-19); that partially covers the $\mathrm{NH} 2$ terminal region and the rod domain; and the major hot spot (exons 43-60), respectively $[10,13]$.

The molecular study revealed a number of different deletions (for details refer to results). The majority of deletions were found at the major hotspot (exon 4552) whether single or multiple. Several investigators around the world pursued the general strategy of deletion analysis presented in this study, which ranged from $45 \%-65 \%$. Frequency as low as $43 \%$ in a Japanese study [19], 37\% in a Chinese study of 41 cases [20], $41 \%$ in a Russian study of 119 cases [3] were reported. One of the biggest studies was pvrsued by Chamberlain et al., 1992 on 745 subjects from several centers

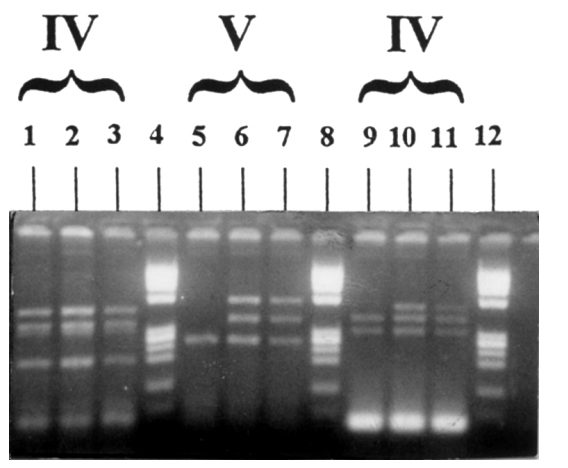

Fig. 2(b). Illustrates 3 multiplexes showing three bands each when normal. Mutiplex IV: three bands in lanes (1)-(3). Mutiplex V: one band and two absent bands (deletions: exon 45 and 51) in lane (5) three normal bands in lanes (6) and (7). Mutiplex IV: one absent band (deletion: exon 48) in lane (9) three normal bands in lanes (10) and (11). The Phi X marker are in (a) lanes (4) (8), (b) (4), (8), (12). The patient's deletion ranging from exon $45-51$ is present in (a) lanes (1), (5), (9), (b) (1), (5), (9).

in the USA screening 9 exons only. The frequency reported was $46 \%$. Relatively higher frequencies were recorded: $63 \%$ in an Italian study [9], 62.5\% in a German study [17], 64\% in an Indian study [2] and 62.3\% in a Brazilian study [21] and 55\% in the present Egyptian study. Such ethnic disparity does not seem to involve genetic pressure, but rather relates to differences in numbers of cases studied as well as numbers and locations of exons analyzed in various populations. It became quite obvious that the more exons analyzed the higher frequency of dystrophin deletions detected, regardless of the ethnic background. For example, numbers of the studied exons in populations displaying low deletion frequencies ranged from 8-15 exons. While in populations with higher deletion frequencies, the number of exons studied ranged from 18-30 exons. In the present study, $55 \%$ of the studied cases had exon 
deletions within both minor and major hot spots with the majority of deletions at exon 45 (23.6\% of which $10.9 \%$ were single exon deletions). In conclusion the deletions found were variable and no specific ethnic differences in respect to deletions could be attributed from this study.

The employment of the 18 exon analysis strategy in this study was designed to be cost effective. When compared with the Indian study [2], where 27 exons were analyzed, only 5 single deletions (one at exon 22, three at exon 52 and one at exon 55) out of a total of 160 cases $(3 \%)$ are expected to be not detectable using the 18 exon analysis indicating that the present method would detect approximately $97 \%$ of deletions in the Indian series with only $2 / 3$ of the cost when planning to launch a nationwide program. Nearly all the 49 deletions in the Indian study including exons $46(6.8 \%)$ and $49(10.8 \%)$ were identified among multiple deletions with the exception of only 2 single deletions at exon $49(0.7 \%)$. These results indicate that inclusion of separate primer sets for amplification of exons 46 and 49 adds little to the value of the 18 primer set strategy employed in the present study. Such a screening method is recommended for identifying the genetic background of a population.

\section{References}

[1] S. Abbs, S.C. You and S. Clark et al., A convenient multiplex PCR system for the detection of dystrophin gene deletions. A comparative analysis with cDNA hybridization shows mistypings by both methods, J. Med Genet. 28 (1991), 304-311.

[2] M. Baneryee and I.C. Verma, Are there ethnic differences in deletion in the dystrophin gene? Am J Med Genet 68 (1997), 152-157.

[3] V.S. Baranov, V.N. Gorbunova, O.V. Mal gsheva and O.V. Artemycva et al., Dystrophin gene analysis and prenatal diagnosis of Duchenne muscular dystrophy in Russia, Prenat Diagn 13 (1993), 323-333.

[4] A.H. Beggs, M. Koenig, F.M. Boyce and L.M. Kunkel, Detection of $98 \%$ of DMD/BMD gene deletions by polymerase chain reaction, Hum Genet. 86 (1990), 45-48.

[5] M.H. Brooke, G.M. Fenichel and R.C. Griggs, Duchenne muscular dystrophy. Patterns of clinical progression and effects of supportive therapy, Neurology 39 (1989), 475-481.

[6] K. Bushby and D. Gardner-Medwin, The Clinical, Genetic and dystrophin characteristics of Becker muscular dystrophy. I Natural History, J. Neurol 240 (1993), 98-104.
[7] C.P. Camphell, Three muscular dystrophies. Loss of cytoskeleton-extracellular matrix linkage, Cell 80 (1995), 675-679.

[8] J.S. Chamberlain, J.R. Chamberlain and F.G. Fenwick et al., Diagnosis of Duchenne and Becker muscular dystrophy by polymerase chain reaction. A multicenter study, JAMA 267 (1992), 2609-2615.

[9] A.E. Covone, F. Caroli and G. Romeo, Screening Duchenne and Becker muscular dystrophy Patients for deletion in 30 exons of dystrophin gene by three multiplex PCRs, Am J Hum Genet 51 (1992), 675-677.

[10] J.T. Den-Dunnen, P.M. Grootscholten, E. Bakker and L.A. Blonden et al., Topography of the DMD gene, FiGE and cDNA analysis of 194 cases reveals 115 deletions and 13 duplications, Am J Hum Genet 45 (1989), 835-847.

[11] A.E.H. Emery, Population frequency of inherited neuromuscular disease: A world survey, Neuromuscular Disorders 1 (1991), 19-29.

[12] K. Matsumora and K.P. Champbell, Dystrophin-glycoprote in complex: Its role in the molecular pathogenesis of muscular dystrophies, Muscle nerve 17 (1994), 2-15.

[13] K. Matsumura, A.H.M. Buighes, M. Maro and F.M.S. Tome et al., Immunohistochemical analysis of dystrophin - associated proteins in Becker/Duchenne muscular dystrophy with huge in-frame deletions in the NH2-terminal and rod domains of dystrophin, J. Clin. Invest. 93 (1994), 99-105.

[14] S.A. Miller, D.D. Dykes and H.F. Polesky, A simple procedure for salting out extracting DNA from human nucleated cells, Nucleic Acids Research 16 (1988), 1215-1219.

[15] A.P. Monaco, C.J. Bertelson and S. Liechti-Gallati, An explanation for the phenotypic differences between patients bearing partial deletions of the DMD locus, Genomics 2 (1988), 90-95.

[16] R. Mountford, PCR techniques for deletion, linkage and mutation analysis in Duchenne/Becker muscular dystrophy, in: Molecular Diagnosis of Genetic Diseases, ed., Rob Elles, 1996, pp. 17-35.

[17] Niemann - S. Seyde, R. Slomski and F. Rininslaad et al., Molecular genetic analysis of 67 Patients with Duchenne/Becker muscular dystrophy, Hum Genet 90 (1992), 65-70.

[18] R.G. Roberts, A.J. Coffey, M. Bobrow and D.R. Bentley, Exon structure of the human dystrophin gene, Genomics 16 (1993), 536-538.

[19] H. Tsukamoto, K. Inui, H. Fukushima and Nishigaki et al., Molecular study of Duchenne and Becker muscular dystrophies in Japanese, J. Inherited Metab. Dis. 14 (1991), 819824.

[20] R.C. Yang, S.L. Yang, S.H. Jin and L.L.W. Chen et al., Detection of dystrophin gene deletion in Chinese Duchenne / Becker muscular dystrophy Patients utilizing multiplex polymerase chain reaction, Kaohsiung J Med Sci 10 (1994), 1-8.

[21] M. Zatz, D. Sumita, S. Campiotto and M. Canovas et al., Paternal inheritance or different mutation in maternally related patients occurs in about $3 \%$ of Duchenne Familial cases, Am J Med Genet 78(4) (1998), 361-365. 


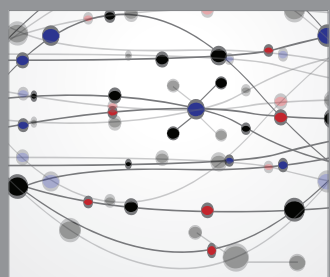

The Scientific World Journal
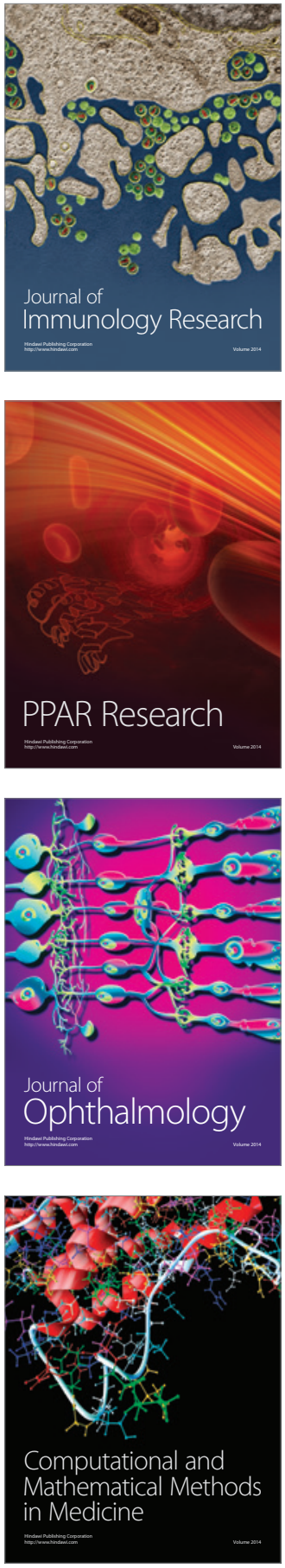

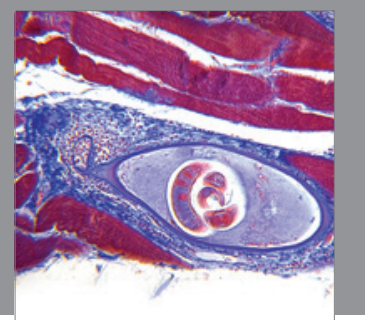

Gastroenterology

Research and Practice
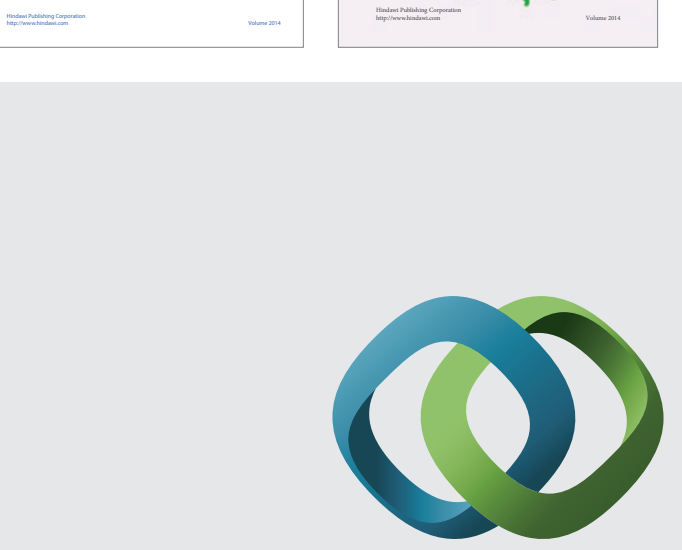

\section{Hindawi}

Submit your manuscripts at

http://www.hindawi.com
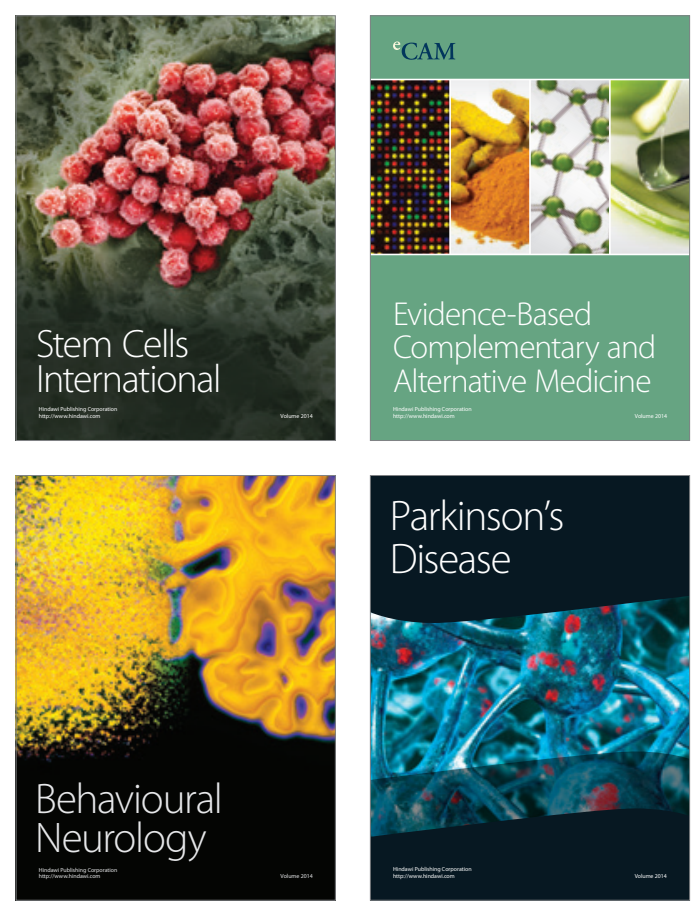

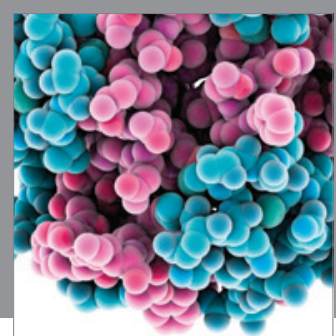

Journal of
Diabetes Research

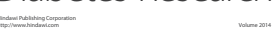

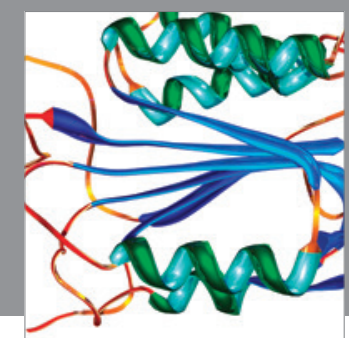

Disease Markers
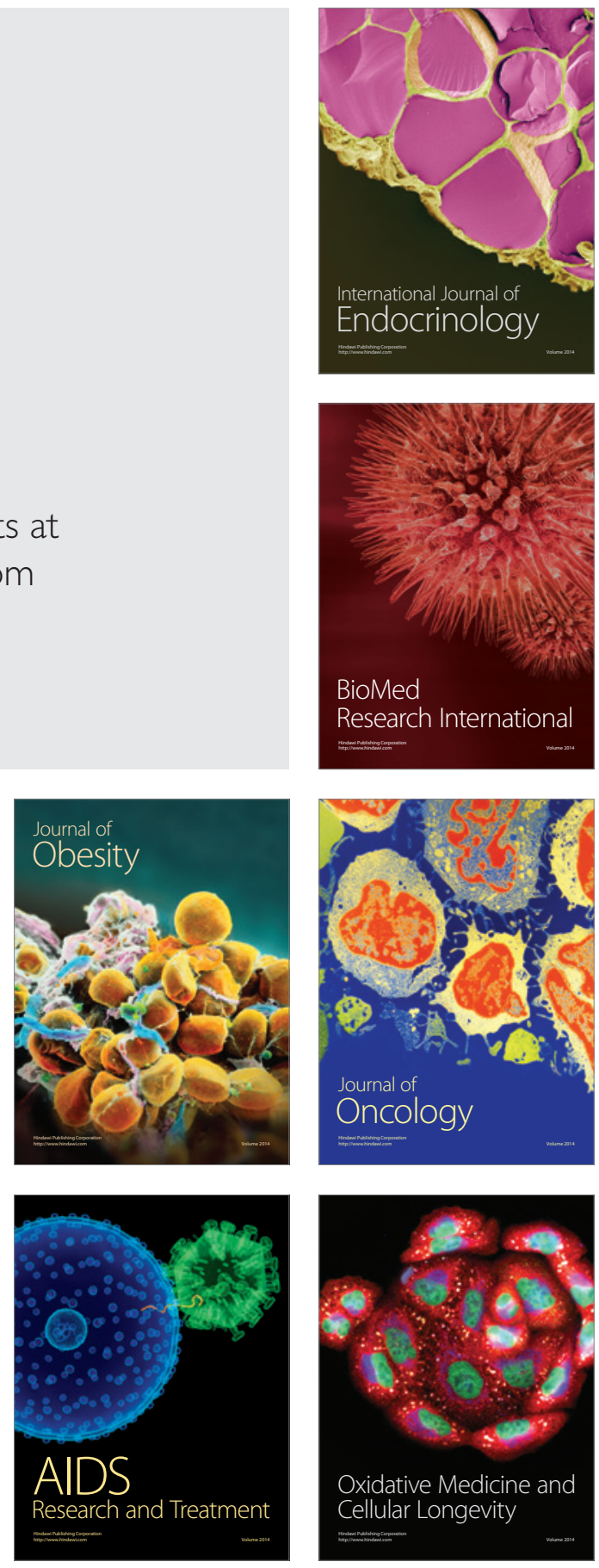